\title{
Long-term Outcomes of One Stage Surgery Using Transanal Colorectal Tube for Acute Colorectal Obstruction of Stage II/III Distal Colon Cancer
}

\author{
Yusuke Okuda, $\mathrm{MD}^{1,2}$ \\ Tomonori Yamada, MD, PhD ${ }^{3}$ \\ Yoshikazu Hirata, MD, PhD² \\ Takaya Shimura, MD, $\mathrm{PhD}^{1}$ \\ Ryuzo Yamaguchi, MD, PhD ${ }^{4}$ \\ Eiji Sakamoto, MD, PhD ${ }^{5}$ \\ Satoshi Sobue, MD, PhD² \\ Takahiro Nakazawa, MD, $\mathrm{PhD}^{3}$ \\ Hiromi Kataoka, MD, $\mathrm{PhD}^{1}$ \\ Takashi Joh, MD, PhD'
}

\section{Purpose}

Since oncological outcomes of transanal colorectal tube (TCT) placement, an endoscopic treatment for colorectal cancer (CRC) with acute colorectal obstruction (ACO), remain unknown, this study analyzed long-term outcomes of TCT placement for stage II/III CRC with ACO.

\section{Materials and Methods}

Data were retrospectively reviewed from consecutive patients with distal stage II/III CRC who underwent surgery between January 2007 and December 2011 at two Japanese hospitals. One hospital conducted emergency surgery and the other performed TCT placement as the standard treatment for all CRCs with ACO. Propensity score (PS) matching was used to adjust baseline characteristics between two groups.

\section{Results}

Among 754 patients with distal stage II/III CRC, 680 did not have ACO (non-ACO group) and 74 had ACO (ACO group). The PS matching between both hospitals identified 234 pairs in the non-ACO group and 23 pairs in the $\mathrm{ACO}$ group. In the non-ACO group, the surgical quality was equivalent between the two institutions, with no significant differences in overall survival (OS) and disease-free survival (DFS). In the ACO group, the rate of primary resection/anastomosis was higher in the TCT group than in the surgery group $(87.0 \%$ vs. $26.1 \%, p<0.001)$. No significant differences were noted between the surgery and the TCT groups in OS (5-year OS, 61.9\% vs. 51.5\%; $p=0.490$ ) and DFS (5-year DFS, $45.9 \%$ vs. 38.3\%; $p=0.658$ ).

\section{Conclusion}

TCT placement can achieve similar long-term outcomes to emergency surgery, with a high rate of primary resection/anastomosis for distal stage II/III colon cancer with ACO.
Correspondence: Takaya Shimura, MD, PhD Department of Gastroenterology and Metabolism, Nagoya City University Graduate School of Medical Sciences, 1-Kawasumi, Mizuho-cho, Mizuho-ku, Nagoya 467-8601, Japan

Tel: 81-52-853-8211

Fax: 81-52-852-0952

E-mail: tshimura@med.nagoya-cu.ac.jp

\section{Key words}

Acute colorectal obstruction, Bridge to surgery, Colorectal neoplasms, Emergency surgery,

Transanal colorectal tube 


\section{Introduction}

Colorectal cancer $(\mathrm{CRC})$ is the third most common malignancy and the fourth leading cause of cancer-related death worldwide [1]. Acute colorectal obstruction (ACO) occurs in $8 \%-29 \%$ of CRC patients [2,3], and this complication requires urgent treatment because of its potential to cause lethal damage, such as perforation, colonic necrosis, and septic shock. In addition, ACO results in high morbidity and mortality in both the short term and the long term [3-5]. Emergency surgical decompression is the gold standard treatment for ACO; however, the morbidity and mortality of emergency surgery are higher than that for elective surgery, in patients without ACO. According to the surgical protocol, two strategies can be used for emergency surgery for ACO, one-stage surgery (primary resection and anastomosis) and staged surgery (an initial procedure, during which a stoma for decompression is created, followed by a later procedure to perform primary resection and stoma closure).

The treatment strategy for ACO is determined on the basis of tumor location. As we previously described, one-stage open surgery is generally performed for right-sided ACO [6], whereas the ideal strategy for left-sided ACO remains controversial. Since the rate of primary resection/anastomosis is lower for left-sided ACO than for right-sided ACO [6-9], preoperative colorectal decompression has become the main treatment for left-sided ACO. Non-surgical treatments such as placement of a self-expanding metallic stent (SEMS) or transanal colorectal tube (TCT) are alternatives to conventional surgical colostomy. Preoperative SEMS or TCT placement is referred to as a bridge to surgery (BTS) to achieve curative primary $\mathrm{CRC}$ resection.

SEMS placement enables control of ACO inflammation and safe one-stage surgery $[10,11]$. Initially, SEMS placement was expected to function as an alternative BTS, and its use had spread widely. Recent meta-analyses, which comprised randomized controlled trials (RCTs), have demonstrated disappointed results where SEMS placement as a BTS enabled primary anastomosis without colostomy, but did not improve short-term mortality [12-14]. In addition, some RCTs revealed that SEMS placement tended to have detrimental effects on disease-free survival (DFS) and recurrence [15-17]. Accordingly, routine use of SEMS are not currently recommended in the curative BTS setting [18].

TCT placement is another alternative endoscopic decompression treatment for $\mathrm{ACO}$, and the authors have previously described the efficacy of TCT placement [19] along with previous studies [20-26]. We have also shown that TCT placement enabled a high frequency of laparoscopic elective surgery [19]. However, no studies have reported the longterm outcomes of TCT placement for CRC with ACO. Long- term outcomes are the most important factor in the treatment of stage II/III CRC in the curative setting [6,18].

Hence, the current study conducted a propensity scorematched analysis and aimed to analyze long-term outcomes of TCT placement for stage II/III distal CRC with ACO in comparison with emergency surgery.

\section{Materials and Methods}

\section{Study setting and patient population}

Consecutive patients with CRC who underwent surgery between January 2007 and December 2011 were retrospectively collected using the computerized databases at two Japanese affiliate hospitals with an interexchange of endoscopists and surgeons: Kasugai Municipal Hospital, Kasugai, Japan (K hospital) and Nagoya Daini Red Cross Hospital, Nagoya, Japan (R hospital). The K hospital performed emergency surgery and the R hospital performed TCT placement as the standard first treatment for ACO, which is due to difference of a director's policy at each institution.

Patients with distal CRCs (distal CRC was defined as CRC located from the transverse colon to the rectum) with pathological stage II/ III were enrolled in this study, after the exclusion of patients with right-sided CRC located in the cecum and the ascending colon because TCT placement is available for transverse colon to the rectum. Patients with distal CRC were divided into two groups: $\mathrm{ACO}$ and non-ACO groups. ACO was diagnosed on the basis of abdominal computed tomography findings as described previously [19].

\section{Surgery}

Surgery can be classified as one-stage surgery and staged surgery. One-stage surgery denotes primary CRC resection with anastomosis. Staged surgery creates a stoma for decompression, and tumor resection and stoma closure are performed later. Open surgery includes open colectomy, open colostomy, and laparoscopic-assisted colectomy (LAC) followed by open colectomy.

In the surgery group, all patients with stage II/III distal CRC with ACO underwent urgent surgery at the $\mathrm{K}$ hospital within 24 hours of admission. Emergency surgery for obstructed CRC was performed by gastroenterological surgeons; therefore, the techniques used for resection depended on the attending surgeon's experience and the patient's specific condition. 


\section{Transanal colorectal tube}

TCT placement was performed as described previously and the Dennis Colorectal Tube (Covidien, Tokyo, Japan) was used as the TCT [19]. The Dennis colorectal tube is $120 \mathrm{~cm}$ long and $22 \mathrm{Fr}$ in diameter. A flexible tapered tip is attached to the distal end of the tube. The TCT was placed over a guide wire that was passed through the stricture under fluoroscopic and endoscopic guidance and irrigated using $>2 \mathrm{~L}$ warm tap water after reduction of intestinal fluid. Following the achievement of technical success, a plain abdominal radiograph was performed after 24 hours of TCT insertion to confirm the TCT position and TCT-induced decompression. The obstructed colon was irrigated with $0.5 \mathrm{~L}$ warm tap water daily until elective surgery. After the colorectal obstruction was relieved, enema examination of the proximal colon was performed to rule out synchronous carcinoma. After $\sim 2$ weeks of TCT placement and after clinical improvement, patients underwent elective surgery. All TCT procedures were performed by endoscopists, and this procedure could be done at any time, 24 hours a day, throughout the year at the $\mathrm{R}$ hospital. Patients were assessed by the surgeon within 2-3 days after TCT placement to plan the elective surgery.

\section{Definitions}

ACO was defined as a situation with colon ileus requiring emergency decompression. Pathological stage was determined on the basis of pathological findings after surgery, according to the seventh edition of the Union for International Cancer Control (UICC) tumor-node-metastasis (TNM) classification [27]. Technical success was defined as TCT placement through the stricture or creation of a stoma. Clinical success was defined as the achievement of patient status in which elective surgery could be performed following decompression. Hospital stay was measured as days of hospitalization for ACO; however, a second period of hospitalization was added to the first period for patients with colostomy who underwent a second surgery for closure. Shortterm mortality was defined as the death rate within 30 days after the first treatment for decompression of colorectal distension. Overall survival (OS) was measured from the first day of treatment until death or the last day of follow-up. DFS was defined as the time from the first day of treatment to recurrence, death, or the last day of follow-up (depending on which event occurred first).

\section{Outcomes}

This study primarily aimed to assess oncological outcomes of TCT placement in the curative setting. Accordingly, we compared the long-term outcomes of OS and DFS between the TCT group and the surgery group in patients with distal stage II/III CRC with ACO. To validate equivalent surgical quality for CRC between the two hospitals, we analyzed OS and DFS for patients with distal stage II/III CRC without ACO. We also analyzed short-term outcomes of TCT placement and emergency surgery for distal stage II/ III CRC with ACO. This study conducted an intention-to-treat analysis: all patients in the R hospital were included in the TCT group even if TCT placement was failed.

\section{Statistical analysis}

We estimated the propensity score (PS) with a logistic regression model, including six factors (age, sex, tumor location, stage, R status, adjuvant chemotherapy). We randomly matched between two groups one by one, using the nearest neighbor method within a caliper of width of 0.05 of the standard deviation of the logit of the PS. Data were analyzed using the Mann-Whitney U test, Student's t test, chi-square test, and Fisher exact probability test, as appropriate. KaplanMeier curves were constructed to analyze OS and DFS, and differences between the two groups were compared with a log-rank test. All statistics were calculated using SPSS statistics, ver. 23 (IBM Corp., Armonk, NY). A p-value $<0.05$ from two tailed tests was considered significant.

\section{Ethical statement}

The present study complied with the STROBE statement [28]. The study protocol was approved by institutional review board of Kasugai Municipal Hospital (IRB No. 275) and Japanese Red Cross Nagoya Daini Hospital (IRB No.1165) (Title: comparison between TCT placement and emergency surgery for stage II/III CRC with ACO), and was conducted according to the ethical guidelines of the 1975 Declaration of Helsinki (6th revision, 2008).

\section{Results}

\section{Patient selection}

Among 983 patients with stage II / III CRC who underwent surgery, 324 were treated in the $\mathrm{K}$ hospital, and 659 received treatment in the R hospital. After the exclusion of 229 patients with right-sided CRC, 754 patients with distal stage II/III CRC from the two hospitals were classified into four subgroups: 234 patients at the K hospital, the non-ACO group; 27 patients at the K hospital, the ACO group; 446 patients at the R hospital, the non-ACO group; and 47 


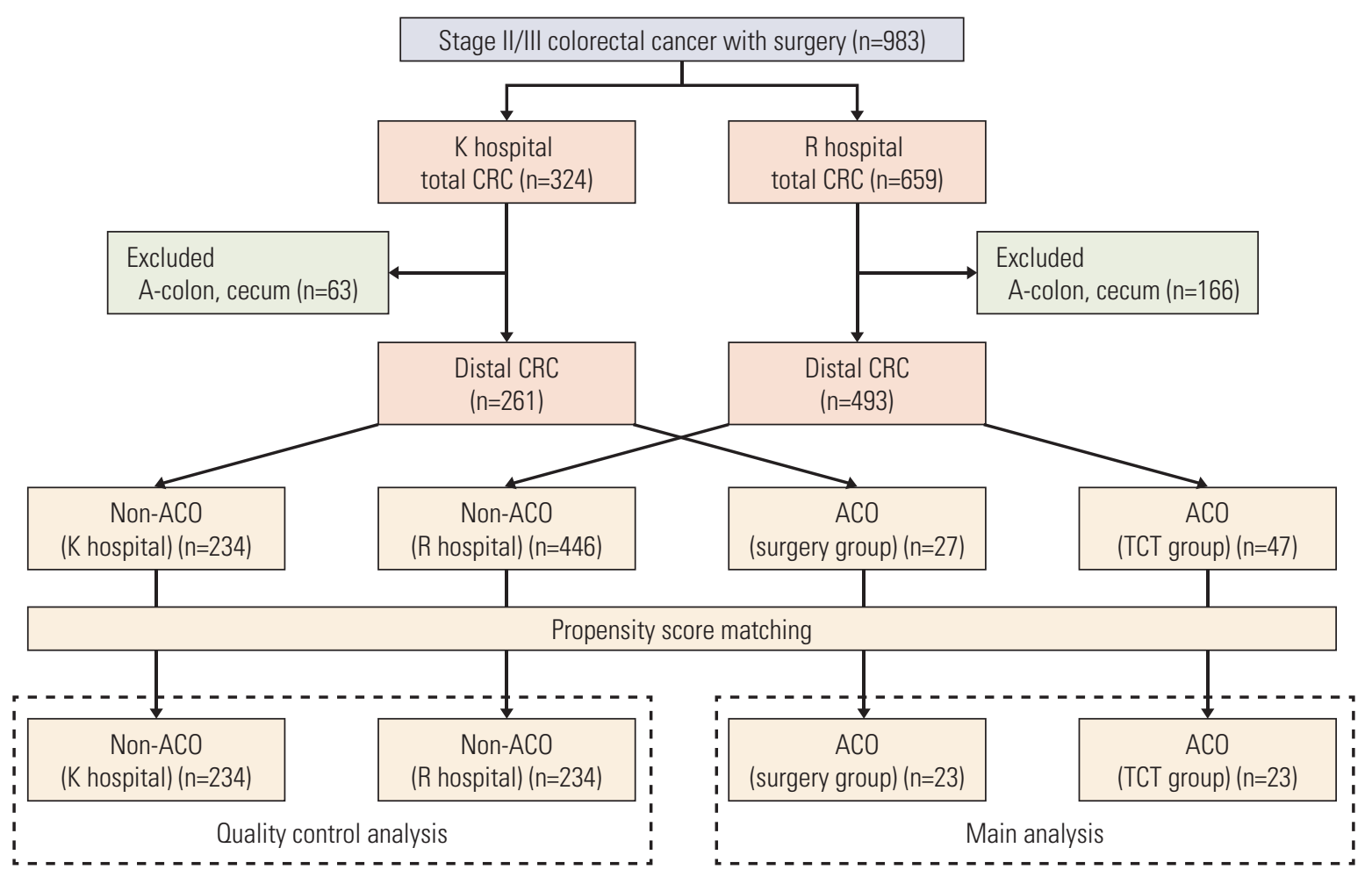

Fig. 1. Consort diagram. The Kasugai Municipal Hospital, Kasugai, Japan (K hospital) applied emergency surgery and the Nagoya Daini Red Cross Hospital, Nagoya, Japan (R hospital) applied transanal colorectal tube (TCT) as the standard treatment for colorectal cancer (CRC) with acute colorectal obstruction (ACO). A-colon, ascending colon.

patients at the R hospital, the ACO group. After one-by-one PS matching, 234 pairs of both hospitals were selected in the non-ACO group and 23 pairs of both hospitals were selected in the ACO group.

Data from the non-ACO groups at both hospitals were used to analyze surgical quality control, and data from the ACO groups at both hospitals were examined for the main analysis (Fig. 1).

\section{Patient characteristics}

Characteristics of patients in both the non-ACO and ACO groups at both hospitals are presented in Table 1. In the nonACO group, some differences were found between two hospitals before PS matching: significantly more patients received adjuvant chemotherapy in the $\mathrm{K}$ hospital (65.0\%) than in the R hospital $(46.2 \%)(\mathrm{p}<0.001)$; tumor location and the R status also showed borderline significant. Baseline characteristics were well balanced after PS matching. Only one patient in the $\mathrm{R}$ hospital received neoadjuvant chemoradiotherapy for rectal cancer.

In the ACO group, no significant differences were found for all parameters between the two hospitals before PS matching; however, the number of patients who received adjuvant chemotherapy displayed a higher tendency in the surgery group (K hospital) than in the TCT group ( $\mathrm{R}$ hospital) $(63.0 \%$ vs. $44.7 \%$; $\mathrm{p}=0.13)$, with a similar tendency in the non-ACO group. The PS matching achieved almost equivalent baseline characteristics between two groups in the ACO group. No rectal cancers were included in the ACO group after PS matching.

Of the 23 patients in the TCT group, 21 underwent TCT placement, and two with right-sided transverse colon cancer underwent transnasal long tube placement.

\section{Quality control analysis}

The 5-year OS in the total study cohort, including both hospitals, was $85.7 \%$ in patients with stage II CRC and $72.8 \%$ in patients with stage III CRC.

As shown in Fig. 2, we investigated the OS and DFS in the PS-matched non-ACO group for quality control analysis. No significant differences were found for OS (5-year OS, K hospital vs. R hospital, $83.2 \%$ vs. $79.0 \%$; $\mathrm{p}=0.30$ ) or DFS (5-year 


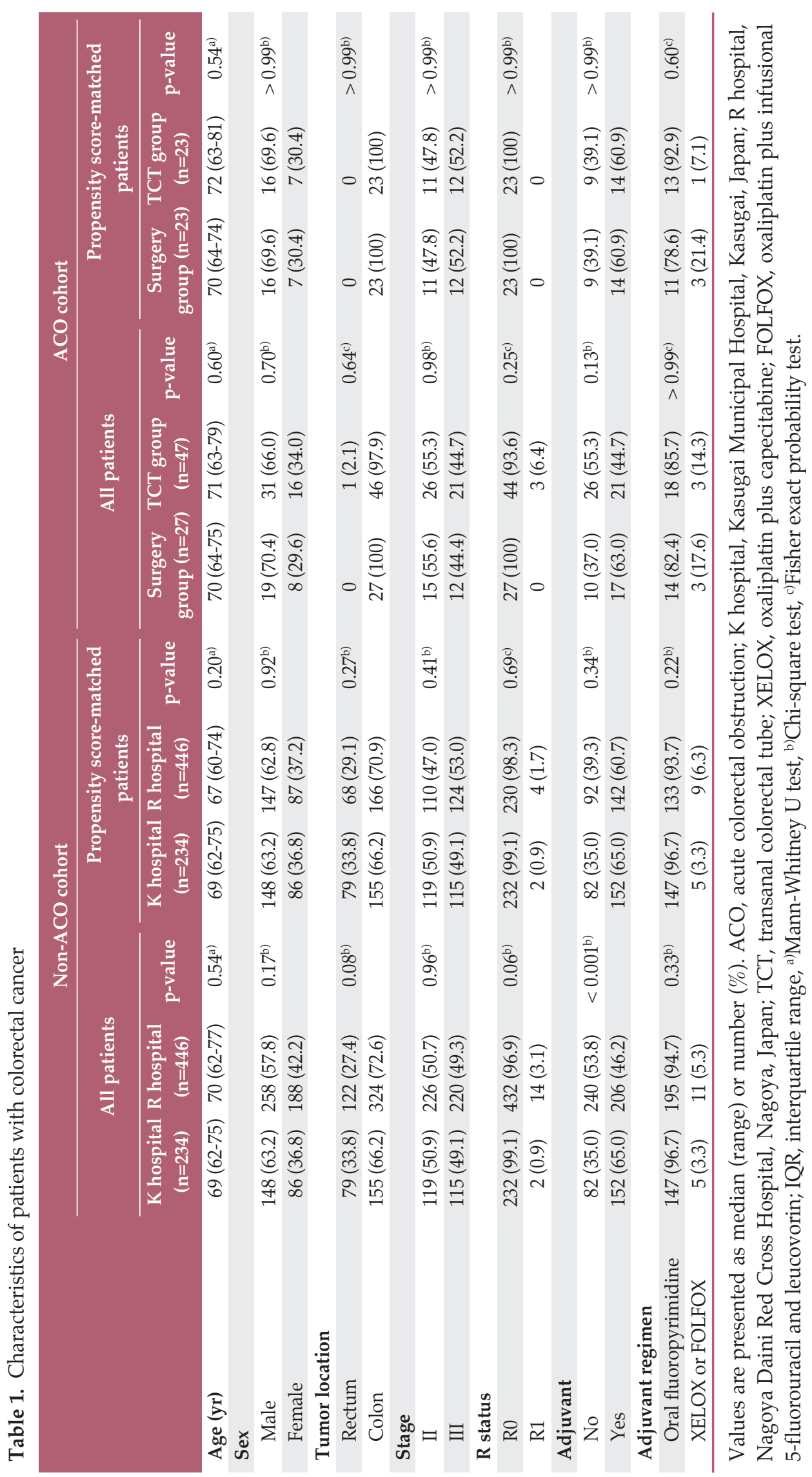



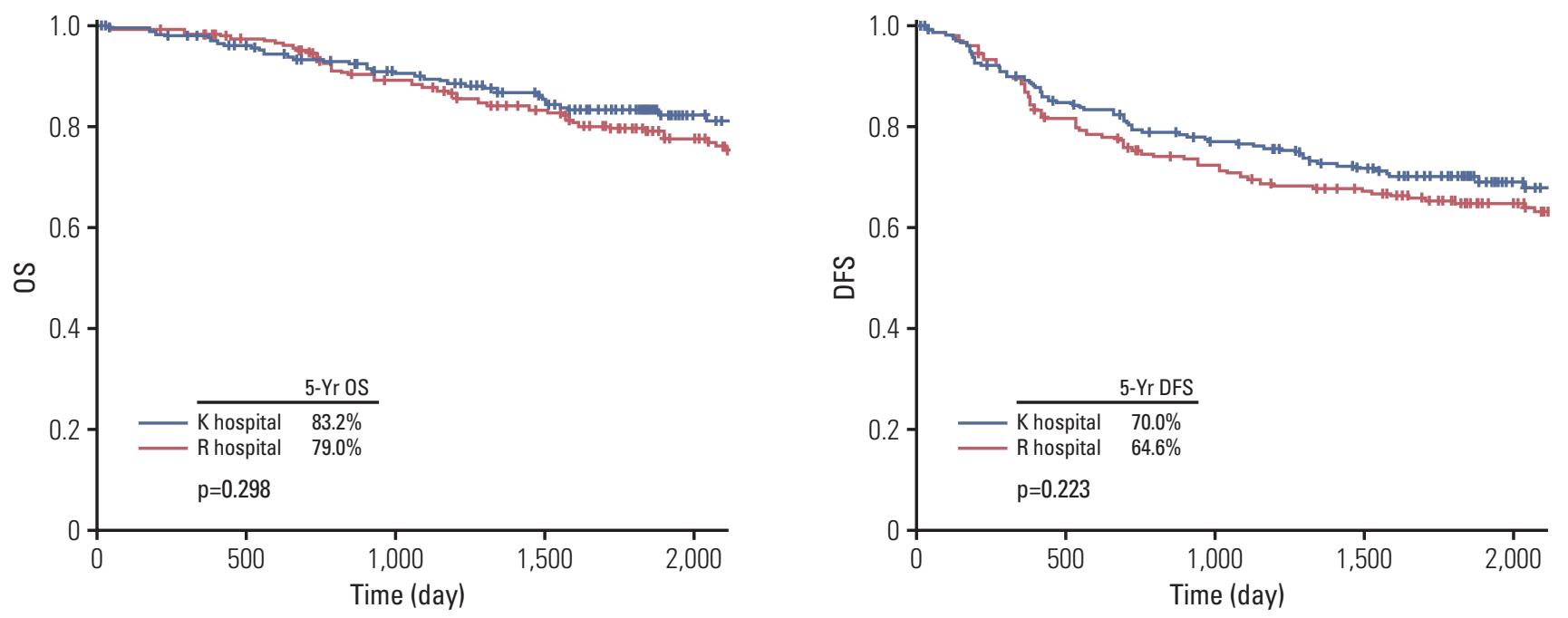

Fig. 2. Overall survival (OS) (A) and disease-free survival (DFS) (B) for stage II / III colorectal cancer without acute colorectal obstruction. K hospital, Kasugai Municipal Hospital, Kasugai, Japan; R hospital, Nagoya Daini Red Cross Hospital, Nagoya, Japan.

Table 2. Short-term outcome

\begin{tabular}{|c|c|c|c|}
\hline & $\begin{array}{c}\text { Surgery group } \\
\text { (K hospital) (n=23) }\end{array}$ & $\begin{array}{c}\text { TCT group } \\
\text { (R hospital) }(n=23)\end{array}$ & p-value \\
\hline Technical success & $23 / 23(100)$ & $23 / 23(100)$ & $>0.99^{a)}$ \\
\hline Clinical success & $23 / 23(100)$ & $22 / 23(95.7)$ & $>0.99^{a)}$ \\
\hline Hospital stay (day) & $30(16-40)$ & $36(27-44)$ & $0.09^{b)}$ \\
\hline Anastomosis leakage & $0 / 23(0.0)$ & $2 / 23(8.7)$ & $0.49^{\mathrm{a})}$ \\
\hline Short-term mortality & $1 / 23(4.3)$ & $0 / 23(0.0)$ & $>0.99^{\mathrm{a})}$ \\
\hline Primary resection/anastomosis & $6 / 23(26.1)$ & $20 / 23(87.0)$ & $<0.001^{\text {a) }}$ \\
\hline Overall stoma & $17 / 23(73.9)$ & $3 / 23(13.0)$ & $<0.001^{\text {a) }}$ \\
\hline Permanent stoma & $2 / 23(8.7)$ & $2 / 23(8.7)$ & $>0.99^{\mathrm{a})}$ \\
\hline LAC & $0 / 23(0.0)$ & $13 / 23(56.5)$ & $<0.001^{\text {a) }}$ \\
\hline
\end{tabular}

Values are presented as number (\%) or median (IQR). K hospital applied emergency surgery and R hospital applied TCT as the first-line standard treatment for CRC with ACO. K hospital, Kasugai Municipal Hospital, Kasugai, Japan; TCT, transanal colorectal tube; R hospital, Nagoya Daini Red Cross Hospital, Nagoya, Japan; LAC, laparoscopy-assisted colectomy; IQR, interquartile range. ${ }^{\mathrm{a}}$ Fisher exact probability test, ${ }^{\mathrm{b}}$ Mann-Whitney $\mathrm{U}$ test.

DFS, K hospital vs. $\mathrm{R}$ hospital, $70.0 \%$ vs. $64.6 \%$; $\mathrm{p}=0.22$ ) between the two institutions, thereby validating comparable surgical quality between the two institutions.

\section{Main analysis}

The short-term outcomes of emergency surgery and TCT placements for the PS-matched ACO group are shown in Table 2 . In the surgery group, the technical and clinical success rates were both $100 \%(23 / 23)$. In the TCT group, the technical success rate was $100 \%$ (23/23), but the clinical success was $95.7 \%$ (22/23) because one patient required urgent surgery owing to tumor perforation. No significant differences were found for the technical and clinical success rates between the surgery and TCT groups. As expected, the rate of primary resection/anastomosis was higher $(87.0 \%$ vs. $26.1 \%, \mathrm{p}<0.001)$, and the overall stoma rate was lower in the TCT group than in the surgery group $(13.0 \%$ vs. $73.9 \%$, $\mathrm{p}<0.001)$. The TCT group achieved a significantly higher rate of LAC compared to the surgery group $(0 \%$ vs. $56.6 \%$, 

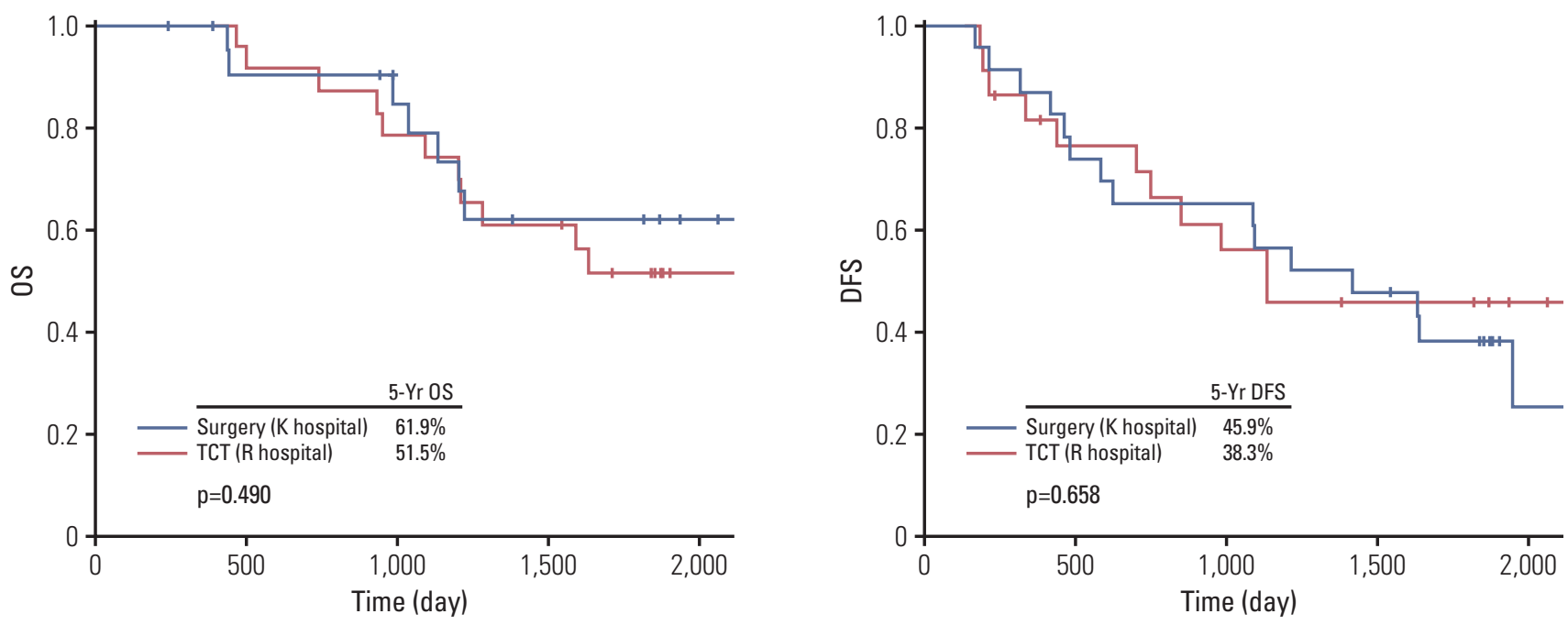

Fig. 3. Overall survival (OS) (A) and disease-free survival (DFS) (B) for stage II/ III colorectal cancer with acute colorectal obstruction. Surgery group comprised patients with emergency surgery at Kasugai Municipal Hospital, Kasugai, Japan (K hospital) and transanal colorectal tube (TCT) group did patients with TCT at Nagoya Daini Red Cross Hospital, Nagoya, Japan (R hospital).

$\mathrm{p}<0.001)$. No significant differences were found for hospital stay, anastomosis leakage, short-term mortality, and permanent stoma between the two procedures.

The long-term outcomes of emergency surgery and TCT placement for the PS-matched ACO group are shown in Fig. 3. No significant differences were noted between the two groups in OS (5-year OS [surgery vs. TCT], 61.9\% vs. 51.5\%; $\mathrm{p}=0.49$ ) or DFS (5-year DFS [surgery vs. TCT], $45.9 \%$ vs. $38.3 \%$; $=0.66)$.

\section{Discussion}

The present study is the first to analyze the long-term outcomes of TCT placement for patients with stage II/III CRC with ACO, in comparison with emergency surgery. The results of this study indicated that TCT placement can achieve similar long-term outcomes to those of emergency surgery and that the technique enabled one-stage surgery with a high rate of primary resection/anastomosis for patients with stage II/III distal colon cancer with ACO. Despite satisfactory decompression for ACO, TCT placement has not been widely used, and its efficacy and safety have not been adequately assessed. The results of the current study demonstrated the long-term efficacy of TCT placement as a BTS and its short-term efficacy.
Since emergency surgery for ACO frequently requires staged surgery and is accompanied by higher risks of mortality and morbidity compared to elective surgery for CRC without ACO [4], the development of alternative methods of conservative management for ACO is desirable. To overcome these issues, SEMS placement has been developed as a BTS for CRC with ACO; however, SEMS placement has not been able to replace emergency surgery owing to its lack of benefit in terms of short-term outcomes and the lack of longterm evidence reported by several RCTs and meta-analyses $[6,18]$. Additionally, SEMS were found to induce more frequent occurrence of tumor ulceration, perineural invasion, and lymph node invasion compared to surgery in a previous case-control study [29], which may be detrimental to oncological outcomes. Hence, another alternative treatment is desirable for ACO management.

A TCT is a transanal drainage tube developed for colorectal decompression treatment, which enables one-stage surgery $[20,22]$. The authors have previously reported that TCT placement also enabled the performance of elective LAC [19]. TCT placement is expected to result in much less damage to the circumference of the tumor than SEMS placement, with an equivalent drainage effect. Moreover, TCT placement might be more cost-effective than SEMS placement [23]. However, there are few data for TCT placement in comparison to SEMS placement, and there are no long-term survival data regarding its role in curative treatment of CRC with ACO. 
With the goal of analyzing long-term outcomes of TCT placement, we compared survival data for patients with stage II/III CRC after treatment between two institutions because emergency surgery is routinely conducted for CRC with $\mathrm{ACO}$ at the $\mathrm{K}$ hospital, while TCT placement is routinely performed for CRC with ACO at the R hospital. Since surgeons and endoscopists in these two institutions belong to the same department and are occasionally exchanged between the two institutions, equivalent surgical quality may be expected. To assess comparison biases between the two different institutions, we first conducted PS-matching and then compared survival data for patients with stage II/III $\mathrm{CRC}$ without $\mathrm{ACO}$ between the two institutions as a quality control analysis because of which we confirmed that there were no significant differences in OS and DFS between the two institutions, with equivalent patient characteristics. These results demonstrated comparable patient populations and surgical quality for CRC between the two institutions. Moreover, 5-year OS in the two hospitals was $85.7 \%$ in patients with stage II CRC and $72.8 \%$ in patients with III CRC patients, both of which are within the upper range of the previous results obtained in Japan (Japanese Society for Cancer of the Colon and Rectum) [30]. These results also indicate that our study is convincing and can be extrapolated to clinical practice.

Next, we investigated the long-term outcomes of patients with ACO after validation of equivalent surgical quality between the two institutions. Consequently, our study using PS-matched analysis clearly demonstrated that OS and DFS of the patients who underwent TCT placement were similar to those of the patients who underwent emergency surgery. In addition to equivalent long-term outcomes, TCT placement enabled LAC and primary resection/anastomosis without stoma in the BTS setting, and no significant differences were found in any adverse events between surgery and TCT placement. Thus, TCT placement can be considered an alternative to $\mathrm{CRC}$ with $\mathrm{ACO}$ in the BTS setting.
The present study had the following limitations. First, since no rectal cancers were included in the ACO group after PS matching, the evidence from this study would be limited to the colon cancer. Second, this was a retrospective study, and the group of CRC patients with ACO consisted of a relatively small sample size for survival analysis. However, this is the first study to analyze long-term outcomes of TCT placement. Moreover, there are no real comparative studies between TCT placement and surgery for even short-term outcomes: although two studies $[19,26]$ previously compared short-term outcomes of TCT placement with those of surgery, the surgery group was consisted of patients with technical failure of TCT such as perforation in one study [26] and with right-sided colon cancers (contraindication of TCT) in the other study [19], which is inappropriate for a comparative study. Consequently, the current data provides plenty of novel insight. Third, each cohort consisted of patients from different hospitals, which may lead to some biases and imbalances. To overcome this concern, we performed PS-matching analysis and we also have validated that there are no significant differences in OS and DFS for patients with stage II/ III distal CRC without ACO between the two institutions. Despite these limitations, the quality of our study might be much higher than general retrospective study because each hospital applied one pure method without any intentional policies, which can eliminate selection bias for treatment.

In conclusion, this study is the first demonstration that TCT placement can achieve similar long-term outcomes and higher rates of one-stage surgery for distal stage II / III colon cancer with ACO than emergency surgery. Preoperative TCT placement followed by elective one-stage surgery may be an alternative strategy for the curative treatment of patients with distal colon cancer patients with ACO.

\section{Conflicts of Interest}

Conflict of interest relevant to this article was not reported.

\section{References}

1. Ferlay J, Soerjomataram I, Dikshit R, Eser S, Mathers C, Rebelo $\mathrm{M}$, et al. Cancer incidence and mortality worldwide: sources, methods and major patterns in GLOBOCAN 2012. Int J Cancer. 2015;136:E359-86.

2. De Salvo GL, Gava C, Pucciarelli S, Lise M. Curative surgery for obstruction from primary left colorectal carcinoma: primary or staged resection? Cochrane Database Syst Rev. 2004;(2):CD002101.

3. McArdle CS, McMillan DC, Hole DJ. The impact of blood loss, obstruction and perforation on survival in patients undergoing curative resection for colon cancer. Br J Surg. 2006;93: 483-8.

4. Biondo S, Pares D, Frago R, Marti-Rague J, Kreisler E, De Oca J, et al. Large bowel obstruction: predictive factors for postoperative mortality. Dis Colon Rectum. 2004;47:1889-97.

5. Katoh H, Yamashita K, Wang G, Sato T, Nakamura T, Watanabe M. Prognostic significance of preoperative bowel obstruction in stage III colorectal cancer. Ann Surg Oncol. 2011;18: 
2432-41.

6. Shimura T, Joh T. Evidence-based clinical management of acute malignant colorectal obstruction. J Clin Gastroenterol. 2016;50:273-85.

7. Sjo OH, Larsen S, Lunde OC, Nesbakken A. Short term outcome after emergency and elective surgery for colon cancer. Colorectal Dis. 2009;11:733-9.

8. Lee YM, Law WL, Chu KW, Poon RT. Emergency surgery for obstructing colorectal cancers: a comparison between rightsided and left-sided lesions. J Am Coll Surg. 2001;192:719-25.

9. Tan KK, Sim R. Surgery for obstructed colorectal malignancy in an Asian population: predictors of morbidity and comparison between left- and right-sided cancers. J Gastrointest Surg. 2010;14:295-302.

10. Ng KC, Law WL, Lee YM, Choi HK, Seto CL, Ho JW. Selfexpanding metallic stent as a bridge to surgery versus emergency resection for obstructing left-sided colorectal cancer: a case-matched study. J Gastrointest Surg. 2006;10:798-803.

11. Martinez-Santos C, Lobato RF, Fradejas JM, Pinto I, OrtegaDeballon P, Moreno-Azcoita M. Self-expandable stent before elective surgery vs. emergency surgery for the treatment of malignant colorectal obstructions: comparison of primary anastomosis and morbidity rates. Dis Colon Rectum. 2002;45: 401-6.

12. Tan CJ, Dasari BV, Gardiner K. Systematic review and metaanalysis of randomized clinical trials of self-expanding metallic stents as a bridge to surgery versus emergency surgery for malignant left-sided large bowel obstruction. Br J Surg. 2012;99:469-76.

13. Ye GY, Cui Z, Chen L, Zhong M. Colonic stenting vs emergent surgery for acute left-sided malignant colonic obstruction: a systematic review and meta-analysis. World J Gastroenterol. 2012;18:5608-15

14. Huang X, Lv B, Zhang S, Meng L. Preoperative colonic stents versus emergency surgery for acute left-sided malignant colonic obstruction: a meta-analysis. J Gastrointest Surg. 2014;18:584-91.

15. Alcantara M, Serra-Aracil X, Falco J, Mora L, Bombardo J, Navarro S. Prospective, controlled, randomized study of intraoperative colonic lavage versus stent placement in obstructive left-sided colonic cancer. World J Surg. 2011;35: 1904-10.

16. Ghazal AH, El-Shazly WG, Bessa SS, El-Riwini MT, Hussein AM. Colonic endolumenal stenting devices and elective surgery versus emergency subtotal/total colectomy in the management of malignant obstructed left colon carcinoma. J Gastrointest Surg. 2013;17:1123-9.

17. Sloothaak DA, van den Berg MW, Dijkgraaf MG, Fockens P, Tanis PJ, van Hooft JE, et al. Oncological outcome of malignant colonic obstruction in the Dutch Stent-In 2 trial. Br J Surg. 2014;101:1751-7.

18. van Hooft JE, van Halsema EE, Vanbiervliet G, Beets-Tan RG,
DeWitt JM, Donnellan F, et al. Self-expandable metal stents for obstructing colonic and extracolonic cancer: European Society of Gastrointestinal Endoscopy (ESGE) Clinical Guideline. Endoscopy. 2014;46:990-1053.

19. Yamada T, Shimura T, Sakamoto E, Kurumiya Y, Komatsu S, Iwasaki $\mathrm{H}$, et al. Preoperative drainage using a transanal tube enables elective laparoscopic colectomy for obstructive distal colorectal cancer. Endoscopy. 2013;45:265-71.

20. Horiuchi A, Nakayama Y, Tanaka N, Kajiyama M, Fujii H, Yokoyama T, et al. Acute colorectal obstruction treated by means of transanal drainage tube: effectiveness before surgery and stenting. Am J Gastroenterol. 2005;100:2765-70.

21. Araki $Y$, Isomoto H, Matsumoto A, Kaibara A, Yasunaga M, Hayashi K, et al. Endoscopic decompression procedure in acute obstructing colorectal cancer. Endoscopy. 2000;32:641-3.

22. Horiuchi A, Maeyama H, Ochi Y, Morikawa A, Miyazawa K. Usefulness of Dennis Colorectal Tube in endoscopic decompression of acute, malignant colonic obstruction. Gastrointest Endosc. 2001;54:229-32.

23. Tanaka T, Furukawa A, Murata K, Sakamoto T. Endoscopic transanal decompression with a drainage tube for acute colonic obstruction: clinical aspects of preoperative treatment. Dis Colon Rectum. 2001;44:418-22.

24. Fischer A, Schrag HJ, Goos M, Obermaier R, Hopt UT, Baier PK. Transanal endoscopic tube decompression of acute colonic obstruction: experience with 51 cases. Surg Endosc. 2008;22: 683-8.

25. Xu M, Zhong Y, Yao L, Xu J, Zhou P, Wang P, et al. Endoscopic decompression using a transanal drainage tube for acute obstruction of the rectum and left colon as a bridge to curative surgery. Colorectal Dis. 2009;11:405-9.

26. Shigeta K, Baba H, Yamafuji K, Kaneda H, Katsura H, Kubochi $\mathrm{K}$. Outcomes for patients with obstructing colorectal cancers treated with one-stage surgery using transanal drainage tubes. J Gastrointest Surg. 2014;18:1507-13.

27. Sobin L, Gospodarowicz M, Wittekind C. TNM classification of malignant tumours. 7th ed. Hoboken, NJ: John Wiley \& Sons, Inc.; 2009.

28. Vandenbroucke JP, von Elm E, Altman DG, Gotzsche PC, Mulrow CD, Pocock SJ, et al. Strengthening the Reporting of Observational Studies in Epidemiology (STROBE): explanation and elaboration. Epidemiology. 2007;18:805-35.

29. Sabbagh C, Chatelain D, Trouillet N, Mauvais F, Bendjaballah $\mathrm{S}$, Browet $\mathrm{F}$, et al. Does use of a metallic colon stent as a bridge to surgery modify the pathology data in patients with colonic obstruction? A case-matched study. Surg Endosc. 2013;27: 3622-31.

30. Watanabe T, Itabashi M, Shimada Y, Tanaka S, Ito Y, Ajioka $Y$, et al. Japanese Society for Cancer of the Colon and Rectum (JSCCR) guidelines 2010 for the treatment of colorectal cancer. Int J Clin Oncol. 2012;17:1-29. 